\title{
Treatment of amiodarone induced hyperthyroidism with potassium perchlorate and methimazole during amiodarone treatment
}

\author{
Louis J M Reichert, Hans A M de Rooy
}

\begin{abstract}
To exploit the antiarrhythmic effect of amiodarone when patients develop the side effect of thyrotoxicosis three patients with hyperthyroidism induced by amiodarone were given simultaneously $1 \mathrm{~g}$ potassium perchlorate a day for $\mathbf{4 0}$ days and a starting dose of $\mathbf{4 0} \mathrm{mg}$ methimazole a day while they continued to take amiodarone. As hyperthyroidism might have recurred after potassium perchlorate treatment was stopped the dose of methimazole was not reduced until biochemical hypothyroidism (raised thyroid stimulating hormone concentrations) was achieved. The patients became euthyroid (free triiodothyronine concentration returned to normal values) in two to five weeks and hypothyroid in $\mathbf{1 0}$ to 14 weeks. One patient became euthyroid while taking $5 \mathrm{mg}$ methimazole a day and $600 \mathrm{mg}$ amiodarone weekly; the two others required substitution treatment with thyroxine sodium while taking $5 \mathrm{mg}$ methimazole or $50 \mathrm{mg}$ propylthiouracil (because of an allergic reaction to methimazole) and 2100 or $1400 \mathrm{mg}$ amiodarone weekly.

Hyperthyroidism induced by amiodarone may be treated with potassium perchlorate and methimazole given simultaneously while treatment with amiodarone is continued.
\end{abstract}

\section{Introduction}

Amiodarone is widely used as an antiarrhythmic drug to control dangerous cardiac irregularities. It has, however, a well recognised propensity to induce thyroid disturbances. ${ }^{1}$ If hyperthyroidism is induced the patient's condition often becomes precarious, with deterioration in the effectiveness of amiodarone, and becomes worse when treatment with amiodarone is stopped. Nevertheless, stopping amiodarone treatment is almost universally accepted before trying to control the hyperthyroidism with antithyroid drugs. As amiodarone contains $37 \cdot 5 \%$ iodine $75 \mathrm{mg}$ iodine per $200 \mathrm{mg}$ tablet) and has an elimination half life of two to three months, however, the thyroid will be overloaded with iodine when the hyperthyroidism occurs and antithyroid drugs might therefore be expected not to have any effect. The commonly observed low uptake of radioactive iodine by the thyroid in amiodarone induced hyperthyroidism prevents the use of iodine-131, and thyroidectomy is often too dangerous to be considered in patients with heart disease and uncontrolled hyperthyroidism. So it seems logical to remove the excess iodine from the thyroid and then start treatment with the antithyroid drug.

Martino et al gave their patients potassium perchlorate and methimazole simultaneously and they became euthyroid in two to five weeks, presumably by the above mechanism. ${ }^{2}$ Martino et al, however, stopped treatment with amiodarone and therefore lost its beneficial antiarrhythmic effect. To be able to continue amiodarone treatment, however, would probably be potentially life saving in a small number of patients. We report on three patients with hyperthyroidism induced by amiodarone who were treated with potassium perchlorate and methimazole while they continued to take amiodarone.

\section{Patients and methods}

The patients were aged 59 (case 1), 71 (case 2), and 67 (case 3) and developed hyperthyroidism during treatment with amiodarone. They all had serious ischaemic heart disease; two patients (cases 1 and 2) had recurrences of dangerous cardiac irregularities, which had previously been responsive only to amiodarone, and developed heart failure. Amiodarone induced hyperthyroidism was thought to be the cause of the deterioration in all three patients, and because of their earlier responsiveness to amiodarone we decided not to stop treatment with amiodarone. Hyperthyroidism was diagnosed 38,37 , and 26 months after amiodarone treatment was started at respective doses of 2100,1400 , and $600 \mathrm{mg}$ weekly. Amiodarone induced hyperthyroidism was diagnosed from the clinical pattern, a decreased thyroid stimulating hormone concentration, a raised free triiodothyronine concentration, and the absence of an image of the thyroid on scanning with technetium- $99 \mathrm{~m}$. Thyroid antibodies were not detected in any of the patients.

Concentrations of free thyroxine and triiodothyronine were measured by radioimmunoassay (Amersham International, Buckinghamshire) and thyroid stimulating hormone concentrations by immunoradiometric assay (Boots, Celltech Diagnostics, Slough, Berkshire). Thyroid antibodies were determined by indirect immunofluorescence at the Central Laboratory of the Blood Transfusion Service in Amsterdam. Laboratory follow up included determination of thyroid stimulating hormone, free triiodothyronine, and free thyroxine concentrations, total blood counts, kidney function, and liver enzyme activities.

In addition to the treatment with amiodarone all three patients received $250 \mathrm{mg}$ potassium perchlorate and $10 \mathrm{mg}$ methimazole, both four times daily. In one patient (case 2) methimazole was stopped after one week and replaced a week later by $100 \mathrm{mg}$ propylthiouracil four times daily because of an allergic reaction. The protocol was approved by the medical ethics committee of this hospital; the three patients gave their informed consent.

\section{Results}

On entry into the study all three patients had a noticeably decreased thyroid stimulating hormone concentration and a highly raised free triiodothyronine

Dr de Rooy. 
concentration. Although amiodarone treatment was continued, treatment with potassium perchlorate and methimazole resulted in the free triiodothyronine concentration returning to normal values after two weeks in one patient and after five weeks in the two others (figure). Free thyroxine, whose conversion to free triiodothyronine is inhibited by amiodarone, ${ }^{3}$ returned to normal values after five to 10 weeks and thyroid stimulating hormone after eight to 12 weeks.
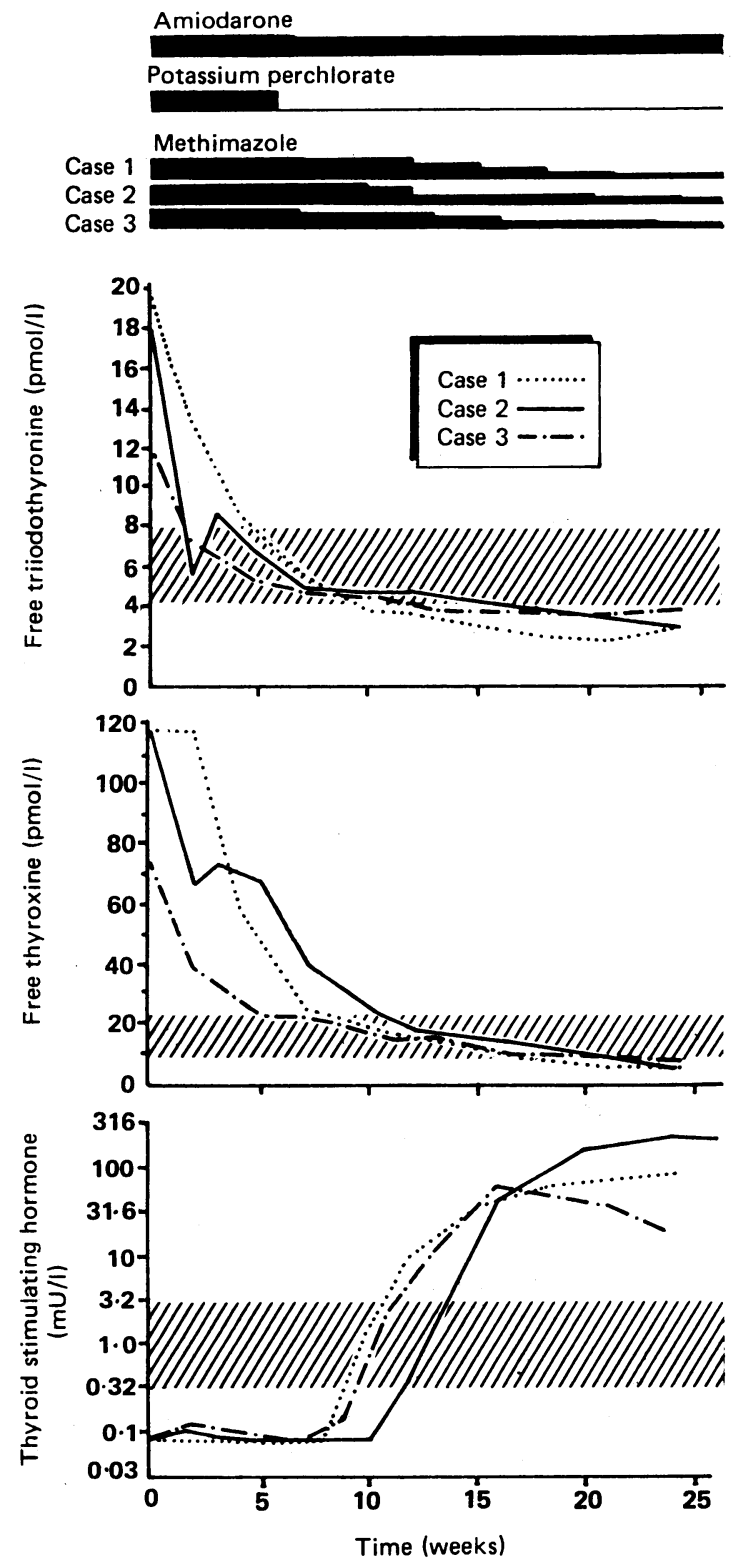

Serial free triiodothyronine, free thyroxine, and thyroid stimulating hormone concentrations in three patients (cases 1-3) with amiodarone induced hyperthyroidism during treatment with amiodarone and $1 \mathrm{~g}$ potassium perchlorate a day for 40 days and methimazole at starting dose of $40 \mathrm{mg}$ a day. Dose of methimazole was gradually reduced but never completely stopped. Shaded areas represent normal ranges

Potassium perchlorate was discontinued after 40 days. After seven to 12 weeks the dose of methimazole was gradually reduced but never completely stopped. During the study no abnormalities were found in the complete blood counts, in the activities of liver enzymes, or in the results of kidney function tests.

One patient (case 3) became euthyroid when given $5 \mathrm{mg}$ methimazole a day and $600 \mathrm{mg}$ amiodarone weekly. The two other patients required substitution treatment with $100 \mu \mathrm{g}$ thyroxine sodium a day while taking $5 \mathrm{mg}$ methimazole (case 1) or $50 \mathrm{mg}$ propylthiouracil (case 2) a day and 2100 or $1400 \mathrm{mg}$ amiodarone weekly. The cardiac irregularities disappeared and the heart failure subsided. All three patients became well and resumed their activities.

\section{Discussion}

Various regimens of treatment have been described for hyperthyroidism induced by amiodarone. Discontinuation of amiodarone treatment lead to spontaneous euthyroidism only after two to eight months. ${ }^{2}$ Martino et al reported good results with a combination of potassium perchlorate and methimazole after amiodarone treatment was discontinued. ${ }^{2}$ Potassium perchlorate may cause the excretion of excess iodine and prevent the uptake of further iodine while methimazole inhibits its incorporation into triiodothyronine and thyroxine.

In 1952 V.yngaarden et al reported that potassium perchlorate interfered with the uptake of inorganic iodine by the thyroid gland in rats. ${ }^{4}$ Because of side effects such as aplastic anaemia and the nephrotic syndrome, which occur predominantly at high doses or with prolonged administration, potassium perchlorate was no longer used for the treatment of hyperthyroidism. ${ }^{5}$ During our study and that of Martino et al the patients received no more than $1 \mathrm{~g}$ potassium perchlorate a day for a maximum of 40 days and laboratory results showed that neither haematological nor renal complications occurred.

Our three patients became euthyroid (free triiodothyronine concentrations returned to normal values) within two to five weeks despite the continuation of amiodarone treatment. The same results were found when amiodarone treatment was discontinued before the hyperthyroidism was treated.

Because of the fear that hyperthyroidism might recur during amiodarone treatment after potassium perchlorate treatment had been discontinued the dose of methimazole was not reduced until biochemical hypothyroidism (raised thyroid stimulating hormone concentrations) was achieved. This may be why our patients rapidly became hypothyroid. As a result we treated two other patients with the same regimen but reduced their dose of methimazole earlier; they became euthyroid and not biochemically hypothyroid within 12 and 18 weeks. Moreover, because of the fear that hyperthyroidism might recur we never completely stopped the antithyroid treatment, not even when the patients' hypothyroidism was sustained (cases 1 and 2). We preferred substitution treatment with thyroxine sodium because we were aware that the excess iodine was prevented from causing a recurrence of the hyperthyroidism only by the persistent (complete or partial) block of peroxidase activity by antithyroid treatment.

We conclude that hyperthyroidism induced by amiodarone may be treated with potassium perchlorate and methimazole during amiodarone treatment.

1 Jonckheer MH, Blockx P, Kaivers R, Wyffels G. Hyperthyroidism as a possible complication of the treatment of ischaemic heart disease with amiodarone. Acta Cardiol (Brux) 1973;28:192-200.

2 Martino E, Aghini-Lambardi F, Mariotti S, et al. Treatment of amiodarone associated thyrotoxicosis by simultaneous administration of potassium perchlorate and methimazole. $f$ Endocrinol Invest 1986;9:201-7.

3 Burger A, Dinichert D, Nicod P, Jenny M, Lemarchand-Béraud T, Vallotton MB. E Dinichert D, Nicod P, Jenny $M$, Lemarchand-Beraud $T$, Valloton MB. Effect of amidarone on serum A drugrion thyronine, thyroxin, and thyrotropin. A drug infuencing peripheral

4 Wyngaarden JB, Wright BM, Ways P. The effect of certain anions upon the accumulation and retention of iodide by the thyroid gland. Endocrinology 1952:50:537-49.

5 Anonymous. Potassium perchlorate and aplastic anaemia [Editorial]. $\mathrm{Br} \mathrm{Med} \mathcal{f}$ $1961 ; \mathrm{i}: 1520-1$

(Accepted 5 April 1989) 